\title{
Pneumatosis Intestinalis: Can We Avoid Surgical Intervention in Nonsurgical Patients?
}

\author{
Ayman Al-Talib ${ }^{a}$ Fahd Al-Ghtani ${ }^{b}$ Roni Munk ${ }^{a}$ \\ aDepartment of Obstetrics and Gynaecology, Division of Gynaecologic Oncology, \\ and ${ }^{b}$ Department of Radiology, McGill University, Montreal, Canada
}

\section{Key Words}

Pneumatosis intestinalis · Conservative management

\begin{abstract}
Pneumatosis intestinalis (PI) is the presence of gas within the wall of the gastrointestinal tract and represents a tremendous spectrum of conditions and outcomes, ranging from benign diseases to abdominal sepsis and death. It is seen with increased frequency in patients who are immunocompromised because of steroids, chemotherapy, radiation therapy, or AIDS. PI may result from intraluminal bacterial gas entering the bowel wall due to increased mucosal permeability caused by defects in bowel wall lymphoid tissue. We present a case of PI who was treated conservatively and in whom PI resolved completely and we present a literature review of conservative management. It is not difficult to make a precise diagnosis of PI and to prevent unnecessary surgical intervention, especially when PI presents without clinical evidence of peritonitis. Conservative treatment is possible and safe for selected patients. Awareness of these rare causes of $\mathrm{PI}$ and close observation of selected patients without peritonitis may prevent unnecessary invasive surgical explorations.
\end{abstract}

\section{Introduction}

Pneumatosis intestinalis (PI) is the presence of gas within the wall of the gastrointestinal tract [1] and is not a diagnosis but a radiographic finding that results from an underlying pathologic process. The significance of PI depends on the nature and severity of the underlying condition. Therefore, PI represents a tremendous spectrum of conditions and outcomes, ranging from benign diseases to abdominal sepsis and death [2].

Pneumatosis is found secondary to mucosal disruption presumably due to overdistension; it is often linear or cystic in appearance, seen with increased frequency in patients who are immunocompromised because of steroids, chemotherapy, radiation 
therapy, or AIDS. In these cases, pneumatosis may result from intraluminal bacterial gas entering the bowel wall due to increased mucosal permeability caused by defects in bowel wall lymphoid tissue. Clinical and imaging findings are important in the differentiation of this transient pneumatosis from fulminant life-threatening causes in this subset of patients. The presence of pneumoperitoneum is the result of a perforation of the gastrointestinal tract in over $90 \%$ of PI patients [3]. In this condition, the signs and symptoms of peritoneal irritation are almost always present and generally the patients require prompt surgical intervention. However, on rare occasions, pneumoperitonitis can occur without any gastrointestinal leakage [4].

\section{Case Report}

A 75-year-old Caucasian lady was diagnosed with stage IIB endometroid adenocarcinoma in 2005. She underwent total abdominal hysterectomy, bilateral salpingo-oophorectomy and pelvic and paraaortic lymph node dissection; she refused adjuvant radiotherapy and was subsequently treated with 4 cycles of a chemotherapeutic regimen consisting of carboplatin and paclitaxel. The patient was unable to tolerate the full treatment course since she developed gastrointestinal bleeding following the fourth dose, so her chemotherapeutic medications were discontinued. The patient was admitted to hospital in March 2006 with increasing breathing difficulties and worsening ascites, at which point the was found on imaging to have recurrence and spread of her initial cancer. She had developed multiple peritoneal implants and pulmonary metastases, so the decision was made to start her on ribosomal doxorubicin $\left(\right.$ Caelyx $\left.{ }^{\circledR}\right)$, of which she received 3 cycles, the last one in May 2007. She presented one week after her third cycle of chemotherapy to the emergency department because she had been vomiting excessively for two consecutive days. She had had a moderate amount of abdominal discomfort associated with increasing ascites gradually worsening over the week prior to admission. She had not had any flatus for 2 days prior to her arrival. The patient was admitted for observation, and it was thought that her symptoms were likely attributable to her last dose of Caelyx ${ }^{\circledR}$ that she had received a few days prior. In any respect, the patient was kept fasting in case she had some small bowel obstruction. She underwent a CT scan of the pelvis and abdomen which demonstrated a large loculated ascites, causing an obstruction to the gastric outlet at the level of the mesenteric vessels with distension of the duodenum and stomach, which were representative of extensive pneumatosis (fig. 1 ). In addition, there was a fairly extensive amount of portal air and free air around the stomach which was highly suggestive of small bowel perforation. Clinically there were no signs of peritonitis and bowel sounds were absent, and the decision was made to treat the patient conservatively since she was not a surgical candidate given the extent of her disease. Octreotide (a long-acting somatostatin analogue) $100 \mu \mathrm{g}$ s.c. q $12 \mathrm{~h}$ was given, metronidazole $500 \mathrm{mg}$ i.v. $\mathrm{q} 8 \mathrm{~h}$, and a nasogastric tube was placed in the stomach and her ascites was drained by paracentesis for comfort measures. A week later she improved clinically and a repeated CT scan of the pelvis and abdomen showed complete resolution of pneumatosis (fig. 2).

\section{Discussion}

Many theories have been suggested in the understanding of the pathogenesis of PI. It is considered that PI sometimes occurs in patients with malignancies during a period of leukocytopenia and may be caused by intestinal mucosal damage due to myelosuppressive agents and immunosuppression from prednisolone [5]. Clinical and imaging findings are important in the differentiation of this transient pneumatosis from fulminant life-threatening causes in this subset of patients. PI, when found incidentally and without signs of peritonitis, is a benign and self-limited condition [6]. The literature contains a number of reports of PI, however few series which described conservative management and outcome. We found 110 patients treated conservatively by different modalities; in 98 patients (89\%) PI resolved while 11 patients (11\%) died. Table 1 summarizes conservative treatment in PI [5, 7-16].

The largest series of conservative management was reported by Morris et al. [16]. 52 patients were treated by nonoperative management consisting of nasogastric tube 
decompression and bowel rest. In 48 patients PI was resolved and 3 patients died; of the 3 patients who died, 1 developed new-onset diabetic ketoacidosis with acute respiratory distress syndrome, and the other 2 had complications of graft-versus-host disease following bone marrow transplant. Knechtle et al. [7] in 1990 examined the management and outcomes of 27 patients who presented with a variety of clinical manifestations and etiologies of their PI. They concluded that patients with clinical evidence of bowel obstruction or ischemia required surgery, the presence of metabolic acidosis, particularly lactic acidosis, and markedly elevated serum amylase suggesting the presence of ischemic bowel. Patients whose preoperative diagnosis suggested ischemia as the primary cause of PI were more likely to have bowel necrosis than those whose PI resulted from bowel obstruction, while those without sepsis or symptoms could be managed conservatively. 16 of 27 underwent laparotomy, with only one negative exploration. 11 patients were treated conservatively, 2 died unrelated to gastrointestinal problems. Of those undergoing surgery, 7 out of 9 had necrotic bowel, with a $75 \%$ mortality rate in the surgery group despite surgical intervention. In the largest series of PI among gynecologic malignancies, Horowitz et al. [8] described the management and outcomes of gynecologic oncology patients with PI. 15 patients with PI were included; 9 patients were managed surgically while 6 were managed conservatively. Pain and tenderness at presentation prompted surgical intervention. No other sign or symptom was significant. Of the 6 patients managed conservatively, 3 (50\%) died within 1 week of diagnosis. Survival for the others was 2, 4, and 6 months. Of the 9 patients managed surgically, 6 (67\%) died, 4 within 2 weeks of surgery and the remainder at 2 and 12 months postoperatively. The 3 patients who survived all had surgical intervention and none had radiographic or pathologic evidence of cancer at the time of presentation. Overall mortality at 6 months was 73\% (11/15). Surgical management was associated with prolonged intensive care unit stay, mechanical ventilation, and sepsis. Conservative management consists of bowel decompression by nasogastric tube, parenteral nutrition and antibiotics prophylaxis by several investigators $[5,7,8]$. The use of antibiotics in resolving PI was based on the bacterial theory, which suggests that gas produced by Escherichia coli, Clostridia, and other gas-fermenting organisms is absorbed and retained within the submucosa and the lymphatic. The resolution of pneumatosis with the use of metronidazole for the treatment of bacterial overgrowth supports this theory [17]. Resolution of pneumatosis by high flow oxygen therapy also provides further circumstantial evidence for a bacterial etiology [18]. Tchabo et al. [9] reported a case of metastatic uterine leiomyosarcoma undergoing resection of the pelvic recurrence; her postoperative course was notable for small bowel obstruction and the finding of PI on radiologic studies. The patient developed mild abdominal pain, but no tenderness or fever. She was managed with bowel rest, nasogastric tube decompression, total parenteral nutrition, and broad-spectrum antibiotics. The finding of PI resolved over the ensuing 6 days. Ade-Ajayi et al. [10] also described conservative management including 7 days of intravenous antibiotics and 10 days of intestinal rest supported by parenteral nutrition in 6 children who developed intestinal pneumatosis following bone marrow transplantation; all patients recovered from the acute episode. Octreotide has been shown to improve pseudoobstruction which is sometimes etiologic. Several authors described good response and resolution of pseudoobstruction with the use of octreotide [12, 19-21]. Kobayashi et al. [12] reported a 26-year-old woman with PI who responded poorly to conventional treatment. Subcutaneous administration of octreotide, at a dose of $50 \mu \mathrm{g} /$ day for 3 weeks, relieved symptoms such as nausea and bloating. There was also a marked decrease of intestinal gas accumulation, as documented on X-ray films. Several cases of successful conservative treatment of PI using hyperbaric oxygen have been reported [18, 22-25]. Grieve and Unsworth [14] reported the largest series of hyperbaric oxygen treatment of PI. 2 patients 
died from unrelated causes; the other 6 were reviewed, and of these 1 declined further endoscopy and the other 5 consented. The 11 courses of treatment all resulted in pronounced symptomatic responses. This was followed by 7 early symptomatic recurrences and 4 long-term cures. These 4 remain asymptomatic and cyst-free at a minimum of 4 years to a maximum of 9 years follow-up. Hashimoto et al. [5] described 4 cases accompanied by hematological malignancies during chemotherapy treatment. All suffered from abdominal discomfort. Abdominal X-ray films revealed gas cysts in the intestine. All 4 cases were treated conservatively by decompression and hyperbaric oxygen. 3 patients improved and 1 patient died of sepsis. Togawa et al. [15] also described a favorable outcome with the use of hyperbaric oxygen treatment in 2 cases of IP.

\section{Conclusion}

The clinical presentation of patients with PI can vary from asymptomatic to critically ill. As a result, the significance of PI is closely linked to the clinical context in which it is found. It is not difficult to make a precise diagnosis of PI and to prevent unnecessary surgical intervention, especially when PI present without clinical evidence of peritonitis. Awareness of these rare causes of PI and close observation of selected patients without peritonitis may prevent unnecessary invasive surgical explorations. However, observation is not appropriate for patients who are hemodynamically unstable or whose clinical evaluation suggests a ruptured gastointestinal tract.

Table 1. Summary of conservative treatment in PI

\begin{tabular}{lrllr}
\hline Author & Patients & Treatment & Outcome \\
\cline { 4 - 5 } & & & death & PI resolved \\
\hline Knechtle et al. [7] & 11 & NG + antibiotics & 2 died (no GI cause) & 9 \\
Horowitz et al. [8] & 6 & NG + antibiotics & 3 died & 3 \\
Hashimoto et al. [5] & 4 & NG + hyperbaric oxygen & 1 died of sepsis & 3 \\
Tchabo et al. [9] & 1 & NG + antibiotics + TBN & - & 1 \\
Ade-Ajayi et al. [10] & 6 & NG + antibiotics + TBN & - & 6 \\
Ripamonti et al. [11] & 17 & NG + antibiotics + Oct & - & 17 \\
Kobayashi et al. [12] & 1 & Oct & - & 2 \\
Masterson et al. [13] & 2 & hyperbaric oxygen & - & 6 \\
Grieve et al. [14] & 8 & hyperparic oxygen & 2 died of other cause & 6 \\
Togawa et al. [15] & 2 & hyperbaric oxygen & - & 2 \\
Morris et al. [16] & 52 & NG & 3 & 98 \\
Total & 110 & & 11 & 98 \\
\hline
\end{tabular}

$\mathrm{NG}=$ Nasogastric tube decompression and bowel rest; $\mathrm{TBN}=$ total parenteral nutrition; $\mathrm{Oct}=$ octerotide (a long-acting somatostatin analogue); GI = gastrointestinal. 


\begin{tabular}{r|l|l|l} 
Case Reports $h$ & $\begin{array}{l}\text { Case Rep Gastroenterol 2009;3:286-292 } \\
\text { Dol: 10.1159/000236596 }\end{array}$ & Published online: September 22, 2009 & $\begin{array}{l}\text { O 2009 S. Karger AG, Basel } \\
\text { ISSN 1662-0631 } \\
\text { www.karger.com/crg }\end{array}$ \\
\hline
\end{tabular}

Fig. 1. Massive loculated ascites, extensive gastric pneumatosis and portal venous gas, as well as a small amount of free gas around the stomach.

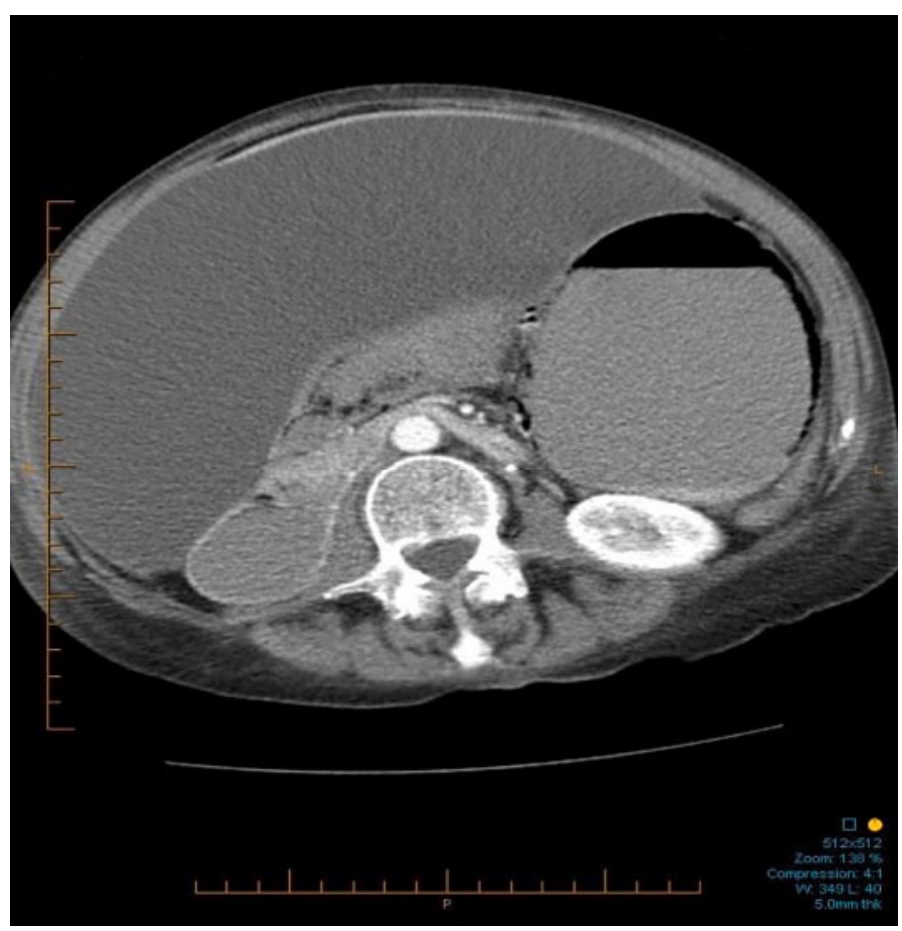

Fig. 2. Marked improvement in portal venous gas and gastric pneumatosis.

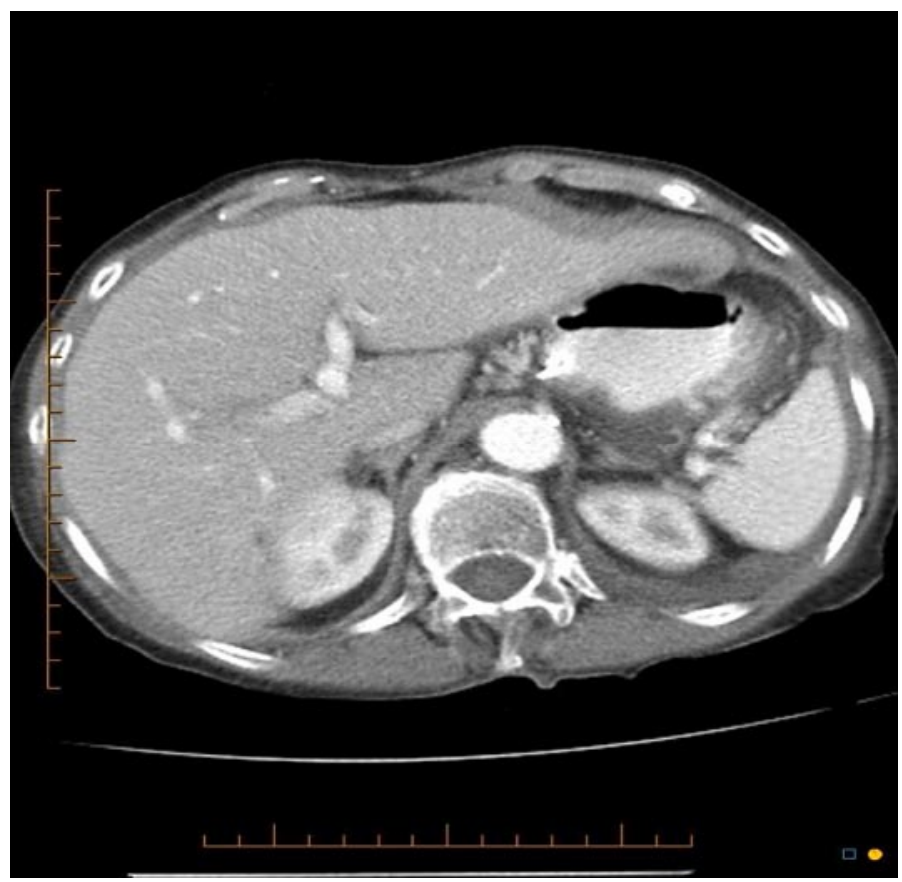




\section{References}

1 Koss LG: Abdominal gas cysts (pneumatosis cystoides intestinorum hominis); an analysis with a report of a case and a critical review of the literature. AMA Arch Pathol 1952;53:523-549.

$\checkmark 2$ St. Peter SD, Abbas MA, Kelly KA: The spectrum of pneumatosis intestinalis. Arch Surg 2003;138:68-75.

-3 McGlone FB, Vivion CG Jr, Meir L: Spontaneous pneumoperitoneum. Gastroenterology 1966;51:393-398.

-4 Omori H, Asahi H, Inoue Y, Irinoda T, Saito K: Pneumoperitoneum without perforation of the gastrointestinal tract. Dig Surg 2003;20:334-338.

-5 Hashimoto S, Saitoh H, Wada K, et al: Pneumatosis cystoides intestinalis after chemotherapy for hematological malignancies: report of 4 cases. Intern Med 1995;34:212-215.

6 Earnest DL, Sleisenger M: Other diseases of the colon and rectum; in Sleisenger M, Fordtran J (eds): Gastrointestinal Disease. Philadelphia, Saunders, 1975, pp 1534-1535.

7 Knechtle SJ, Davidoff AM, Rice RP: Pneumatosis intestinalis. Surgical management and clinical outcome. Ann Surg 1990;212:160-165.

-8 Horowitz NS, Cohn DE, Herzog TJ, et al: The significance of pneumatosis intestinalis or bowel perforation in patients with gynecologic malignancies. Gynecol Oncol 2002;86:79-84.

-9 Tchabo NE, Grobmyer SR, Jarnagin WR, Chi DS: Conservative management of pneumatosis intestinalis. Gynecol Oncol 2005;99:782-784.

10 Ade-Ajayi N, Veys P, Stanton M, Drake DP, Pierro A: Conservative management of pneumatosis intestinalis and pneumoperitoneum following bone-marrow transplantation. Pediatr Surg Int 2002;18:692-695.

-11 Ripamonti C, Mercadante S, Groff L, Zecca E, De Conno F, Casuccio A: Role of octreotide, scopolamine butylbromide, and hydration in symptom control of patients with inoperable bowel obstruction and nasogastric tubes: a prospective randomized trial. J Pain Symptom Manage 2000;19:23-34.

12 Kobayashi T, Kobayashi M, Naka M, Nakajima K, Momose A, Toi M: Response to octreotide of intestinal pseudoobstruction and pneumatosis cystoides intestinalis associated with progressive systemic sclerosis. Intern Med 1993;32:607-609.

13 Masterson JS, Fratkin LB, Osler TR, Trapp WG: Treatment of pneumatosis cystoides intestinalis with hyperbaric oxygen. Ann Surg 1978;187:245-247.

14 Grieve DA, Unsworth IP: Pneumatosis cystoides intestinalis: an experience with hyperbaric oxygen treatment. Aust N Z J Surg 1991;61:423-426.

-15 Togawa S, Yamami N, Nakayama H, Shibayama M, Mano Y: Evaluation of HBO2 therapy in pneumatosis cystoides intestinalis. Undersea Hyperb Med 2004;31:387-393.

16 Morris MS, Gee AC, Cho SD, et al: Management and outcome of pneumatosis intestinalis. Am J Surg 2008;195:679-683.

17 Tak PP, Van Duinen CM, Bun P, et al: Pneumatosis cystoides intestinalis in intestinal pseudoobstruction. Dig Dis Sci 1992;37:949-954.

-18 Holt S, Gilmour HM, Buist TA, Marwick K, Heading RC: High flow oxygen therapy for pneumatosis coli. Gut 1979;20:493-498.

19 Jackson JE, Thomas AMK: The case of the bloated nurse. Br J Radiol 1987;60:721722.

20 Yonemitsu K, Hayakawa M, Takeuchi I, et al: Pneumatosis cystoides intestinalis and pneumoperitoneum complicated with progressive systemic sclerosis: a case report. Nippon Naika Gakkai Zasshi 1988;77:734-735.

21 Fallon RH: Pneumatosis cystoides intestinalis, associated with scleroderma and presenting with pneumoperitoneum. Mo Med 1967;64:117-118.

22 Forgacs P, Wright PH, Wyatt AP: Treatment of intestinal gas cysts by oxygen breathing. Lancet 1973;1:579-582.

23 Gruenberg JC, Batra SK, Priest RJ: Treatment of pneumatosis cystoides intestinalis with oxygen. Arch Surg 1977;112:62-64. 
24 Hoflin F, Van der Linden W: Pneumatosis cystoides intestinalis treated by oxygen breathing. Scand J Gastroenterol 1974;9:427-430.

25 Paw HGW, Reed PN: Pneumatosis cystoides intestinalis confined to the small intestine treated with hyperbaric oxygen. Undersea Hyperb Med 1996;23:115117. 